\title{
Equilibration rates and negative absolute temperatures for ultracold atoms in optical lattices
}

\author{
Akos Rapp, Stephan Mandt, and Achim Rosch \\ Institute for Theoretical Physics, University of Cologne, 50937 Cologne, Germany
}

(Dated: October 23, 2018)

\begin{abstract}
As highly tunable interacting systems, cold atoms in optical lattices are ideal to realize and observe negative absolute temperatures, $T<0$. We show theoretically that by reversing the confining potential, stable superfluid condensates at finite momentum and $T<0$ can be created with low entropy production for attractive bosons. They may serve as "smoking gun" signatures of equilibrated $T<0$. For fermions, we analyze the time scales needed to equilibrate to $T<0$. For moderate interactions, the equilibration time is proportional to the square of the radius of the cloud and grows with increasing interaction strengths as atoms and energy are transported by diffusive processes.
\end{abstract}

PACS numbers: 05.30.Fk,05.30.Jp,05.60.Gg,05.70.Ln,03.75.Nt

The concept of temperature is central in thermodynamics. For most systems, where the energy $E$ has no upper bound, only positive temperatures, $T>0$, are allowed in equilibrium. However, in any system with an upper bound in energy, $T<0$ are possible. In this case, states with higher energy are occupied more likely than states with lower $E$. Negative $T$ and even phase transitions at $T<0$ have been realized in nuclear spin systems [1 3] : antiferromagnetically coupled nuclear spins show ferromagnetic order at $T<0$ where high-energy states are dominantly populated. Such an "inverted" population is also the basis of most lasers.

While negative temperatures lead to many nonintuitive results, all laws of thermodynamics can equally be applied [4]. Figure 1(a) shows schematically the entropy $S$ as a function of energy for a system with a maximal and minimal energy. As $1 / T=\partial S / \partial E$, negative $T$ arises whenever the entropy decreases as a function of energy. One consequence is that a Carnot engine, which operates between two reservoirs with temperatures $T_{1}<0$ and $T_{2}>0$, has an efficiency $\eta$ larger than 1: $\eta=W / Q_{1}=1-T_{2} / T_{1}>1$, where $W$ is the work done and $Q_{1}$ is the heat extracted from the (hotter) reservoir. Usually, $\eta<1$ as the entropy $\Delta S$ extracted from the hot reservoir has to be dumped into the second reservoir and the corresponding heat $T \Delta S$ is lost. If the first reservoir has, however, negative $T$, its energy is lowered when the entropy increases, see Fig. 1(a): heat can be extracted from both systems simultaneously and therefore $\eta>1$.

With quantum-optical methods it is possible to manipulate atoms in an unprecedented way. Atoms in optical lattices allows us not only to realize Hubbard models [5] for bosons [6] and fermions [7, 8], but also to control all parameters with high precision. In the following, we will first show how simple manipulations of such systems can be used to create and detect unambiguously equilibrated quantum states at negative $T$, following partially a proposal of Mosk [9], and then we discuss in the case of fermions the time scales needed for equilibration.

Atoms in sufficiently deep optical lattices can be de- (a)

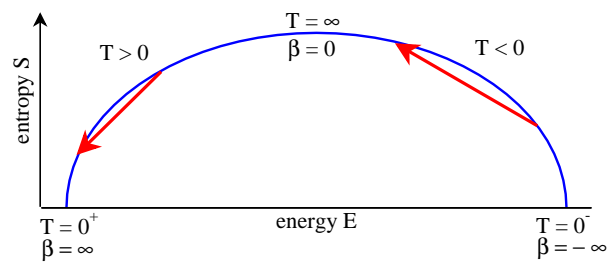

(b)

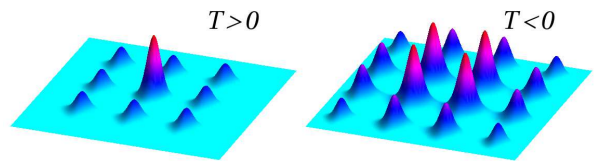

FIG. 1: (color online). (A) Entropy as a function of energy (schematically) for a system with an upper and lower bound of energy. For high energies, $1 / T=\partial S / \partial E$ is negative. Arrows: for two reservoirs with $T>0$ and $T<0$, respectively, one can remove reversibly energy from both reservoirs by reducing the entropy for $T>0$ and increasing it by the same amount for $T<0$. (B) Schematic time-of-flight image of expanding bosons. For $T>0$, repulsive bosons condense at momentum 0 (with higher order peaks from Bragg reflections). For $T<0$, condensates of attractive bosons form in the maxima of the kinetic energy for momenta $( \pm \pi / a, \pm \pi / a, \pm \pi / a)$.

scribed by Hubbard models [5] given by

$$
\begin{aligned}
H_{f}=-J & \sum_{<i j>\sigma}\left(f_{i \sigma}^{+} f_{j \sigma}+\text { h.c. }\right)+U \sum_{i} n_{i \uparrow} n_{i \downarrow} \\
& +V_{0}(t) \sum_{i \sigma} \mathbf{r}_{i}^{2} n_{i \sigma}, \\
H_{b}=-J & \sum_{<i j>}\left(b_{i}^{+} b_{j}+\text { h.c. }\right)+\frac{U(t)}{2} \sum_{i} n_{i}\left(n_{i}-1\right) \\
& +V_{0}(t) \sum_{i} \mathbf{r}_{i}^{2} n_{i},
\end{aligned}
$$

for fermions and bosons, respectively. $U$ is the local interaction, $J$ the tunneling rate and $\sigma=\uparrow, \downarrow$ the hyperfine index for the fermions. $V_{0}(t)$ represents a time-dependent parabolic potential with $n_{i \sigma}=f_{i \sigma}^{+} f_{i \sigma}, n_{i}=b_{i}^{+} b_{i}$. In Eqs. (1-2) higher bands are omitted. As discussed in detail by Mosk [9], typical tunneling rates into such bands 
are exponentially suppressed and negligible.

Negative $T$ in equilibrium is only possible for Hamiltonians bounded from above. Thus, for the models (1.2), $V_{0}<0$ and for bosons also $U<0$ is required. Nevertheless, ultracold atoms have to be prepared with some $V_{0}>0$ initially, and we shall discuss how $T<0$ can be reached from such conditions (preparation of high-energy states in spin-systems is discussed in Ref. [10]).

To get some intuition on negative $T$, note that the equilibrium density matrix, $e^{-H / k_{B} T}$, for a Hamiltonian $H$ at $T<0$ is identical to the density matrix for reversed temperature $\tilde{T}=-T$ and a Hamiltonian $\tilde{H}=-H$. For the models (11]2) therefore considering $T<0$ is equivalent to $\tilde{T}>0$ with parameters $-V_{0},-U$, and most importantly, $-J$. As for a cubic lattice with lattice constant $a$ the sign of $J$ can be absorbed into a shift of all momenta, $k \rightarrow k+Q$ with $Q=(\pi / a, \pi / a, \pi / a)$ [using [11] that $-J \cos (k a)=J \cos (k a+\pi)$ ], the phase diagram of the negative- $U$ Hubbard models for $T<0$ are identical to that of the positive- $U$ Hubbard models if the momenta are shifted in all observables. Most dramatically, bosons will therefore condense at momenta $( \pm \pi / a, \pm \pi / a, \pm \pi / a)$, i.e., in the maxima of the band structure, for $U<0, T<0$ [ see Fig. 1(b)]. Note that $T<0$ bosonic condensates are stable for attractive and unstable for repulsive $U$. For fermions superfluidity can also be reached for $T<0, U>0$ but in this case the fermionic pairs condense at zero momentum as $\sum_{k} f_{k \uparrow}^{+} f_{-k \downarrow}^{+}=\sum_{k} f_{k+Q \uparrow}^{+} f_{-k-Q \downarrow}^{+}$. We remark that it has been shown [12] that for $U \gg J$ one can nevertheless induce a condensate at momentum $Q$ even for fermions in a different nonequilibrium situation. To summarize, the momentum distribution of a Bose-Einstein condensate is probably the best way to detect $T<0$ due to the qualitative difference to the $T>0$ system, and we will quantitatively estimate under what conditions such a state can be reached.

In principle, it is possible to reach $T<0$ without any entropy production by first adiabatically decreasing $J$ until $J=0$, then switching suddenly $U \rightarrow-U$ and $V_{0} \rightarrow-V_{0}$, followed by an adiabatic increase of $J$ until the desired value is reached. In practice this is not a good choice as the timescales for equilibration diverge for $J \rightarrow 0$. Therefore one needs a faster scheme where $J$ remains finite but as little entropy as possible is produced. As a reversal of $V_{0}, U$ and $T$ is formally equivalent to a sudden quench $J \rightarrow-J$ without inverting $T$ (see above), the basic idea is to start from an initial state where $J$ is finite but the kinetic energy is very small, i.e., a Mott or a band insulator.

For bosons we propose the following. The system is (I) prepared in a Mott-insulating state. (II) $J$ is switched off suddenly by increasing the intensity of the optical lattice. This freezes all density-density correlation functions. One waits (III) for a time $t_{\mathrm{w}}$ during which the potential and the interaction are reversed slowly, $V_{0} \rightarrow-V_{0}$ and $U \rightarrow-U$. (IV) $J$ is switched suddenly back to its initial value and (V) the system equilibrates. Finally, (VI) the negative trapping potential and/or the interaction strength $U$ is weakened adiabatically with the goal to reach a superfluid condensate at $T<0$. For fermions, parts of this scheme have been implemented in Ref. [11], where, however, $V_{0}$ was not reversed but set to 0 .

The waiting time $t_{\mathrm{w}}$ allows us to reverse $V_{0}$ and $U$ slowly (manipulation of $U$ requires "slow" changes of magnetic fields in some experimental setups), and more importantly, it leads to a complete dephasing of the kinetic energy, $E_{\text {kin }}$, as has been argued in Ref. [1]]: During $t_{W}$ each site in the lattice collects a different phase due to $V_{0}$. Effectively, therefore $E_{\text {kin }} \propto \sum_{\langle i j\rangle}\left\langle b_{i}^{\dagger} b_{j}\right\rangle$ averages to zero for $t_{W} \gg 1 / \Delta V$, where $\Delta V$ is the potential difference between neighboring sites. Note that for a Mott insulator $E_{\text {kin }}$ vanishes in the center of the trap but is sizable in the outer parts of the cloud where $\Delta V$ is large. Estimates using the parameters of Ref. [6] show that waiting times $t_{\mathrm{w}} \gtrsim 10 \mathrm{~ms}$ are sufficient for a complete dephasing.

For a quantitative estimate of the entropy generated during this sequence, one first needs to know the total energy $E_{\text {tot }}$ after step (V), i.e., before the final equilibration stage. As the kinetic energy is zero after dephasing and the initial density distributions are fully preserved, we obtain $E_{\text {tot }}=-\left(E_{\text {int }}+E_{\text {pot }}\right)$, where $E_{\text {int }}$ and $E_{\text {pot }}$ are the initial interaction and potential energies, respectively. We obtain these variationally using the Gutzwiller wave function for bosons [13] assuming a low- $T$ initial state for a given strength of the confining potential characterized by the dimensionless compression $V_{0} N^{2 / 3} / 6 J$, where $N$ is the number of atoms. This combination appears naturally [8] as the thermodynamic limit in a trap is given by $N \rightarrow \infty$ with $V_{0} N^{2 / 3}=$ const.

Energy conservation allows us to determine the state after equilibration. The corresponding temperature will be negative and large. We therefore used a hightemperature expansion [14] to obtain the thermodynamic potential in the presence of the trap. We found that an expansion up to $4^{\text {th }}$ order in $\beta J$ gives accurate results. For simplicity, we locally approximated the system by a homogeneous one. Corrections to this so-called localdensity approximation [15] vanish with $1 / N^{1 / 3}$ and are therefore tiny for typical atom numbers, $N \sim 10^{5}$. Finally, the (negative) temperature was determined by setting $\left\langle H_{b}\right\rangle=E_{\text {tot }}$ and then the entropy was computed from the high- $T$ expansion.

In Fig. 2 the entropy per boson is shown as a function of the initial compression $V_{0} N^{2 / 3}$ of the cloud for several values of $U$. We also calculated the result without dephasing (dashed lines), obtained in the limit $t_{\mathrm{W}} \rightarrow$ 0 , corresponding to the scheme originally proposed by Mosk [9]. Entropies and $|T|$ are about $40 \%$ higher in this case.

The entropy per boson drops for increasing $\left|V_{0}\right|$ as 


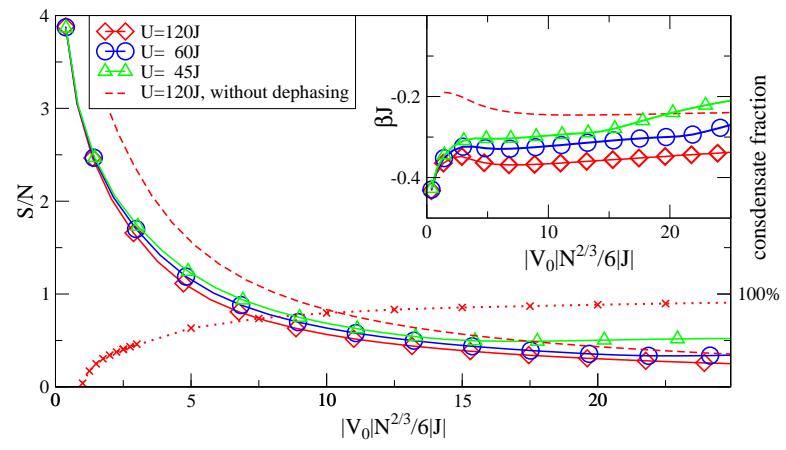

FIG. 2: (color online). Entropy per boson as a function of the initial confinement $V_{0}$, for different initial interactions $U$ after following the steps (I)-(V), see text. Dotted line: Condensate fraction at momenta $( \pm \pi / a, \pm \pi / a, \pm \pi / a)$ for $U=120 \mathrm{~J}$ after a slow decrease of $|U|$ during step (VI). A slightly higher condensate fraction is obtained, if in step (VI) $\left|V_{0}\right|$ is reduced (not shown). Inset: inverse temperatures. Dashed lines: results for $U=120 \mathrm{~J}$ using the protocol suggested by Mosk [9], i.e., without dephasing of the kinetic energy.

larger and larger fractions of the cloud become Mott insulating and thus insensitive to sign changes of $J$. Most importantly, even for moderate compressions and moderate values of $U$ the entropy per boson is well below the critical entropy of an ideal Bose gas in a $d=3$ harmonic trap, $S / N<s_{0}=3.6 k_{B}$. This implies that for realistic parameters, one can expect the formation of the negative$T$ Bose-Einstein condensate after the adiabatic expansion [step (VI)] characterized by the momentum distribution of Fig. $1 \mathrm{~B}$ and by a large condensate fraction. Figure 2 shows the condensate fraction assuming that in step (VI) $|U|$ has been reduced adiabatically to 0 for fixed $V_{0}<0$.

We will now consider spin- $1 / 2$ fermions focusing on dimensions $d=2$ to simplify numerics. With fermions one can reach $T<0$ using a time-independent $U$ starting from a band insulating, rather than a Mott-insulating initial state. We will focus our discussion on the time scales needed to reach $T<0$. To determine the dynamics, we use a numerical solution of the Boltzmann equation in relaxation time approximation, [1]

$$
\partial_{t} f+\mathbf{v}_{\mathbf{k}} \cdot \nabla_{\mathbf{r}} f+\mathbf{F} \cdot \nabla_{\mathbf{k}} f=-\frac{1}{\tau(n, e)}\left(f-f_{0}(n, e)\right),
$$

where $f(\mathbf{r}, \mathbf{k}, t)$ is the occupation probability in phase space and the force term $\mathbf{F}=-\nabla_{\mathbf{r}}\left(V_{0} \mathbf{r}^{2}\right)-U \nabla_{\mathbf{r}} n(\mathbf{r})$ contains the external potential and interaction corrections on the Hartree level. $n=n_{\uparrow}=n_{\downarrow}=n(\mathbf{r}, t)$ and $e=e(\mathbf{r}, t)$ are the local particle densities per spin and energy density, respectively, and $f_{0}(n, e)$ is a Fermi function chosen such that energy and particle number are conserved. In Ref. [11] we have determined the scattering rate $1 / \tau(n, e)$ to reproduce the $e$ - and $n$-dependent diffusion constant of the Hubbard model to order $U^{2}$ (obtained from an independent calculation). Most importantly, $1 / \tau(n, e)$ vanishes for low densities, $1 / \tau(n, e) \propto n$, in the tails of the cloud where scattering is rare. In Ref. [11] the numerics was also compared to experiments.

We first consider an instantaneous quench, $V_{0 i} \rightarrow$ $V_{0 f}=-0.05 V_{0 i}$ for $U=2 J$ and parameters which reflect approximately the experimental conditions of Ref. [11]. Temperature and density profiles (and parameters) are displayed in Fig. 3. Here $T(\mathbf{r}, t)$ is defined as the temperature of a homogeneous reference ensemble in equilibrium with the same energy and particle density.

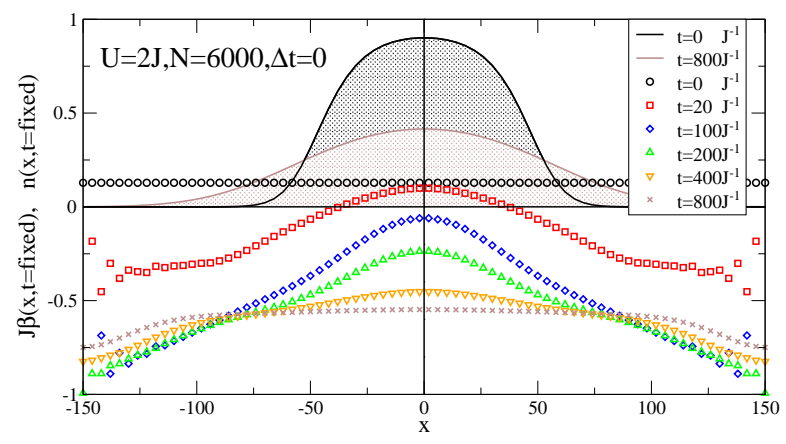

FIG. 3: (color online). Cuts through the density $n$ (lines) and inverse temperature $\beta=1 / k_{B} T(\mathbf{r}, t)$ (symbols) for quenches $V_{0 i} \rightarrow-0.05 V_{0 i}$ at $U=2 J, V_{0} N=60 J$, initial entropy $S / N=1.2 k_{B}$ and $N=6000$ particles per spin.

Because of energy conservation and the upper bound on the kinetic energy, the atomic cloud cannot expand to arbitrary size but equilibrates for $t \rightarrow \infty$. The initial $T>0$ becomes rapidly negative and slowly obtains a homogeneous value (which can be calculated from energy conservation in an independent way). It takes longer to equilibrate in the tails of the cloud, where scattering rates are small and strong Bloch oscillations occur.

Two time scales determine the relaxation mainly. First, $\tau(n, e)$ determines locally the equilibration rate according to Eq. (3) but local interactions do not change the local energy and particle density. Therefore a second time scale describes how long it takes to redistribute particles and energy across the cloud. For not too weak interactions, see below, this transport will be diffusive and not convective as momentum is not conserved and can be efficiently transferred to the optical lattice by umklapp scattering for the relatively high $|T|$ considered here. The corresponding diffusion time is estimated as

$$
\tau_{D} \sim \frac{r^{2}}{D} \sim \frac{U^{2} N}{J^{3}}
$$

where $r$ is the radius of the cloud, $D \sim v^{2} \tau$ the diffusion constant, $v \sim J a$ the typical velocity (for $T \sim J$ ), $1 / \tau \sim$ $n U^{2} / J$ and $n(r / a)^{2} \sim N$. The inset of Fig. 4 displays $1 / T$ in the center of the cloud as a function of $\tau_{D} / t$. It shows that $\tau_{D}$ becomes the relevant time scale for large $N$ and not too small $U$. Furthermore, for $t \ll \tau_{D}$, where the edges of the cloud do not yet play a role, there is a regime, where $\beta \sim 1 / t$ is observed as expected for 
(energy) diffusion in $d=2$. Figure 4 also shows the time $t_{90}(U)$ needed to reach $J \beta_{0}=-0.469$ (90\% of the inverse temperature for $t \rightarrow \infty, U \rightarrow 0$ ). For larger $U$ one gets $t_{90} \sim \tau_{D} \propto U^{2}$ but for small $U, t_{90}$ diverges due to the divergence of the local relaxation time, $\tau \propto$ $1 / U^{2}$. In all cases, relaxation is not very fast, $t_{90}>$ $300 / J$. The fastest relaxation to equilibrium occurs for relatively weak interactions when $U$ is a fraction of the full bandwidth $8 J$.

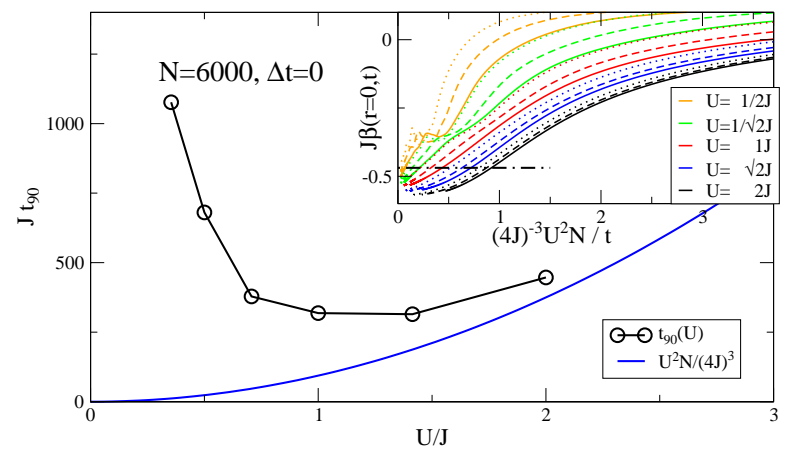

FIG. 4: (color online). Time required to reach $\beta_{0}=0.9 \times$ $\beta_{f}(U \rightarrow 0)$ for $N=6000$. Inset: $\beta(\mathbf{r}=0, t)$ in the center of the trap as a function of rescaled $1 / t$ for various $U$ and system sizes (solid: $N=3000$, dashed: $N=6000$, dotted: $N=12000)$. Dash-dotted line: $J \beta_{0}=-0.469$.

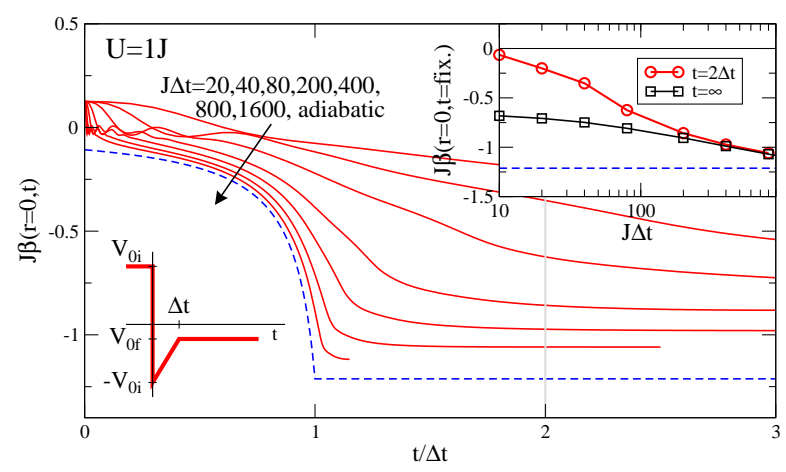

FIG. 5: (color online). The inverse temperature $\beta(\mathbf{r}=0, t)$ in the center of the cloud as a function of $t / \Delta t$ for different opening times $\Delta t, U=J$ and a $V_{0}(t)$ shown schematically in the left inset $\left(V_{0 f}=-0.05 V_{0 i}, V_{0 i} N=60 \mathrm{~J}, N=6000\right)$. Right inset: Inverse temperatures $\beta(\mathbf{r}=0, t=\infty)$ and $\beta(\mathbf{r}=$ $0, t=2 \Delta t)$ as a function of $\Delta t$.

In order to reach $T<0$ with small $|T|$, it is useful to decrease $\left|V_{0}\right|$ slowly to reduce entropy generation and to see whether adiabatic conditions can be realized. We therefore use the protocol shown in the inset of Fig. 5 . after a sudden quench at $t=0, V_{0 i} \rightarrow-V_{0 i},-V_{0}$ is reduced linearly, $V_{0}(t)=-V_{0 i}+\left(V_{0 f}+V_{0 i}\right) t / \Delta t$ for $t<\Delta t$ and $V_{0}(t)=V_{0 f}<0$ for $t>\Delta t$. As shown in Fig. [5], upon increasing $\Delta t$, considerably lower values of $\beta<0$ can be obtained and one approaches the adiabatic limit. Only due to the high entropy assumed for the initial state
$\left(S / N=1.2 k_{B}\right.$, implying $J \beta \approx 1.47$ for $V_{0 i} \rightarrow\left|V_{0 f}\right|$ adiabatically), $|T|$ remains relatively high even for $\Delta t \rightarrow \infty$ where $J \beta \approx-1.21$. The overall entropy production for $\Delta t \rightarrow \infty$ is tiny, $\Delta S / N \approx 0.12 k_{B}$, [c.f. the bosonic case, Fig. 2 as for the large initial $T$, kinetic energies were small. Note that even for $\Delta t=1600 / J$ deviations from the adiabatic behavior are considerable, which shows how difficult it is to reach truly adiabatic conditions. Nevertheless, it is possible to reach $T \approx-2 J$ within a time $200 / J \sim 100 \mathrm{~ms}$ for typical parameters [11].

In our opinion the observation of finite momentum superfluidity, Fig. 1B, is probably the best "smoking gun" signature of $T<0$ in equilibrium. To reach it, it is, however, necessary to switch the interaction $U \rightarrow-U$ using a Feshbach resonance for bosons. We expect that the associated loss processes by three-particle scattering can efficiently be reduced in an optical lattice. An important issue are the time scales needed for local equilibration and - most importantly - redistribution of energy and particles across the system. For fermions we find that relaxation is most efficient for relatively weak interactions. More generally, the long equilibration times arising from the necessity to redistribute energy and particles should be important for all equilibration processes and quenches in inhomogeneous systems both for positive and negative $T$. Here we expect that the equilibration properties of low- $|T|$ bosons differ qualitatively from high- $|T|$ fermions due to the suppression of umklapp scattering for bosons and due to the onset of superfluidity.

We acknowledge discussions with I. Bloch, D. Rasch, E. Demler, and U. Schneider, and financial support by the SFB 608 and SFB/TR 12 of the DFG and the Studienstiftung des deutschen Volkes (S.M.).

[1] E.M. Purcell and R.V. Pound, Phys. Rev. 81279 (1951).

[2] P. J. Hakonen, et. al., Phys. Rev. Lett. 68, 365 (1992).

[3] A. S. Oja and O. V. Lounasmaa, Rev. Mod. Phys. 69, 1 (1997).

[4] N. F. Ramsey, Phys. Rev. 103, 20 (1956).

[5] D. Jaksch, et. al., Phys. Rev. Lett. 81, 3108 (1998).

[6] M. Greiner, et. al., Nature 415, 39 (2002).

[7] R. Jördens, et. al., Nature 455, 204-207 (2008).

[8] U. Schneider, et. al., Science 322, 1520-1525 (2008).

[9] A. P. Mosk, Phys. Rev. Lett. 95, 040403 (2005).

[10] A. S. Sorensen, et. al., , preprint, arXiv:0906.2567

[11] U. Schneider, et. al., , preprint, arXiv:1005.3545

[12] A. Rosch, et. al., Phys. Rev. Lett. 101, 265301 (2008).

[13] W. Krauth, M. Caffarel, and J.-P. Bouchaud, Phys. Rev. B 45, 3137-3140 (1992).

[14] J. Oitmaa, C. Hamer, and W. Zheng: Series expansion methods for strongly interacting lattice models (2006, Cambridge University Press, Cambridge, England, 2006 ) .

[15] I. Bloch, J. Dalibard, W. Zwerger, Rev. Mod. Phys. 80, 885 (2008). 